\title{
A SPLITTING THEOREM FOR ABELIAN-BY-FC-HYPERCENTRAL GROUPS
}

\author{
M. J. TOMKINSON \\ Department of Mathematics, University of Glasgow, Glasgow G12 8QW, UK
}

(Received 17 April, 1998)

\begin{abstract}
We extend splitting theorems due to Zaicev and Duan proving the following result. Let $G$ be a locally soluble $F C$-hypercentral group and let $A$ be a periodic artinian $\mathbb{Z} G$-module. If $A$ has no finite $\mathbb{Z} G$-submodules then any extension $E$ of $A$ by $G$ splits conjugately over $A$.
\end{abstract}

1991 Mathematics Subject Classification. 20F19

1. Introduction. There have been a number of results of the following type: let $G$ be a group with property $\mathfrak{X}$ and $A$ a $\mathbb{Z} G$-module with property $\mathfrak{V}$ then any extension $E$ of $A$ by $G$ splits over $A$ and any two complements are conjugate in $E$. We say that $E$ splits conjugately over $A$.

A number of these results have nilpotency conditions on $G$ and conditions on $A$ which ensure that there are no central factors of $E$ inside $A$ (or the $\mathbb{Z} G$-module $A$ has no trivial $\mathbb{Z} G$-sections). Among these results are those given by Robinson [4] in homological terms. Perhaps the simplest and most striking form is:

Let $G$ be a hypercentral group and $A$ a $\mathbb{Z} G$-module. If $A$ is noetherian and $H_{0}(G, A)=0($ i.e. $[A, G]=A)$ or $A$ is artinian and $H^{0}(G, A)=0\left(\right.$ i.e. $\left.C_{A}(G)=0\right)$ then $H_{n}(G, A)=H^{n}(G, A)=0$ for all $n$.

This implies that any extension of $A$ by $G$ splits conjugately over $A$ as this condition is equivalent to $H^{1}(G, A)=H^{2}(G, A)=0$.

Both the statement of the above result and the methods used by Robinson seem to indicate that this is the best approach when $G$ has a nilpotency condition. However other results of this type have not been successfully put into a homological setting partly because it is not possible to give such a nice description of the conditions on the module $A$. For these results more purely group theoretic methods have been used.

D. I. Zaicev [5-8] considered hypercyclic and hyperfinite extensions of abelian groups with conditions ensuring that the module $A$ had no cyclic, respectively finite, $\mathbb{Z} G$-sections. Z. Y. Duan $[\mathbf{1 , 2}]$ showed that these results could be amalgamated by considering a locally soluble hyper-(cyclic or finite) group $G$ and a noetherian or periodic artinian $\mathbb{Z} G$-module $A$ with no finite $\mathbb{Z} G$-sections. The conclusion was that in these situations any extension $E$ of $A$ by $G$ splits conjugately over $A$.

However, L. A. Kurdachenko has pointed out an error in [1] and [7], the papers dealing with the artinian case. The error occurs in Lemma 1 of [7] where in the case that $A \neq C_{0}$ it is possible that $A=C_{0}+D$ when the argument fails to provide a nonzero $\mathbb{Z} G$-image. The same error was repeated in [1] and also in an earlier version of the present paper.

We aim then to correct and extend the results of Zaicev and Duan in the artinian case proving the following 
Theorem. Let $G$ be a locally soluble FC-hypercentral group and let $A$ be a periodic artinian $\mathbb{Z} G$-module. If $A$ has no finite $\mathbb{Z} G$-submodules then any extension $E$ of $A$ by $G$ splits conjugately over $A$.

Of course, the only difference between this result and Duan's is that we can include free abelian factors of finite rank greater than one in the group $G$. Apart from the error referred to above the parts of the proof which do not involve these factors are very similar to those given by Zaicev [7] and Duan [1] but significant extra work is needed to deal with the finite rank factors. The methods used are therefore derived from those of Zaicev and we make no attempt to find a suitable homological setting for the theorem.

2. Notation and preliminaries. The $F C$-centre of a group $G$ is the characteristic subgroup consisting of all elements having only finitely many conjugates in $G$. It is denoted by $F C(G)$ and, being an $F C$-group, contains a periodic characteristic subgroup with a torsion-free abelian factor group. We can form the upper $F C$-series of $G$ by defining $G_{\alpha+1} / G_{\alpha}=F C\left(G / G_{\alpha}\right)$ and $G_{\beta}=\cup_{\alpha<\beta} G_{\alpha}$, for limit ordinals $\beta$. If $G_{\rho}=G$ for some ordinal $\rho$ then $G$ is said to be FC-hypercentral. By considering normal closures of elements in the periodic part of $F C(G)$ and then of those in the torsion-free factor, we see that an $F C$-hypercentral group has an ascending normal series in which the factors are finite or are free abelian of finite rank generated by the conjugates of a single element.

If $A$ is a $\mathbb{Z} G$-module, we shall say it is periodic if it is periodic as an abelian group. A $\mathbb{Z} G$-image of $A$ is a factor $A / B$, where $B$ is a $\mathbb{Z} G$-submodule of $A$; a $\mathbb{Z} G$ section of $A$ is a $\mathbb{Z} G$-module $B / C$ where both $B$ and $C$ are $\mathbb{Z} G$-submodules of $A$. A $\mathbb{Z} G$-composition factor of $A$ is a $\mathbb{Z} G$-section which is simple (=irreducible) as a $\mathbb{Z} G$ module.

If a subgroup $H$ of $G$ acts trivially on $A$ (that is, $a h=a$, for all $a \in A, h \in H$ ) we say that $A$ is $\mathbb{Z} H$-trivial. If $x \in Z(G)$ and $A$ is a $\mathbb{Z} G$-module then $A(1-x)$ and $C_{A}(x)$ are $\mathbb{Z} G$-submodules and $A(1-x) \cong A / C_{A}(x)$ as $\mathbb{Z} G$-modules. The $\mathbb{Z} G$-modules $C_{A}(x)$ and $A / A(1-x)$ are $\mathbb{Z}<x>$-trivial.

We shall use $[A, H]$ to denote the $\mathbb{Z} H$-submodule $\sum_{h \in H} A(1-h)$. If $H \triangleleft G$, then $[A, H]$ is a $\mathbb{Z} G$-submodule of $A$. Although this commutator notation seems inconsistent with the notation for the action of $\mathbb{Z} G$ on $A$, it is in common use in our situation as later we shall be thinking of the $\mathbb{Z} G$-module $A$ as a normal abelian subgroup of an extension $E$ of $A$ by $G$.

Similarly, we use $C_{A}(H)$ for the fixpoint submodule $\{a \in A: a h=a$, for all $h \in H\}$.

Some of the inconsistency could perhaps be avoided by writing $a^{x}$ rather than ax for the action of $G$ on $A$ and, in fact, we do use this notation later when we are definitely within the extension setting.

Frequently, we shall have a subgroup $G_{1}$ of finite index in $G$ and need to consider the $\mathbb{Z} G$-module $A$ as a $\mathbb{Z} G_{1}$-module. In this situation it is well-known that $A$ is $\mathbb{Z} G$-artinian if and only if it is $\mathbb{Z} G_{1}$-artinian and also that $A$ has no non zero finite $\mathbb{Z} G_{1}$-sections if and only if it has no non-zero finite $\mathbb{Z} G_{1}$-sections.

In the statement of our main theorem we have given the condition that $A$ has no non-zero finite $\mathbb{Z} G$-submodules. This is, in fact, equivalent to $A$ having no non-zero finite $\mathbb{Z} G$-sections as is shown by the following decomposition theorem which has appeared recently. 
Theorem 2.1. (Kurdachenko, Petrenko and Subbotin [3]) Let $G$ be an FChypercentral group and $A$ an artinian $\mathbb{Z} G$-module. Then $A$ has a direct decomposition $A=A^{+} \oplus A^{-}$, where every $\mathbb{Z} G$-composition factor of $A^{+}$is finite and $A^{-}$has no nonzero finite $\mathbb{Z} G$-sections.

This decomposition is usually referred to as an $f$-decomposition of $A$.

3. Proof of the theorem. There are a number of places in the proof where we will have a normal subgroup $G_{1}$ of finite index in $G$ and the $\mathbb{Z} G$-module $A$ will have a nonzero $\mathbb{Z} G_{1}$-image $A / B$ with certain properties. To obtain a nonzero $\mathbb{Z} G$-image in which we can work we use the following canonical construction of a $\mathbb{Z} G$-image which is a direct sum of suitable $\mathbb{Z} G_{1}$-images.

Lemma 3.1. Let $G / G_{1}$ be finite with $T=\left\{g_{1}, \ldots, g_{n}\right\}$ a transversal to $G_{1}$ in $G$. Let $A$ be a $\mathbb{Z} G$-module and $B$ a proper $\mathbb{Z} G_{1}$-submodule of $A$. For each set $J$ of subsets of $\left\{g_{1}, \ldots, g_{n}\right\}$ form $B(J)=\sum_{I \in J}\left(\bigcap_{i \in I} B g_{i}\right)$ and let $B\left(J_{0}\right)$ be a maximal member of the set $\{B(J) \mid B \leq B(J)$ and $B(J) \neq A\}$. Then $B_{0}=\cap_{g \in G} B\left(J_{0}\right) g$ is $a \mathbb{Z} G$-submodule of $A$ and

$$
A / B_{0}=\oplus\left\{A / B\left(J_{0}\right) g \mid g=g_{i_{l}}, \ldots, g_{i_{l}} \text {, some } l \leq n\right\}
$$

Proof. Clearly we can write $B_{0}=B\left(J_{0}\right) g_{1} \cap \ldots \cap B\left(J_{0}\right) g_{n}$. We show, by induction on $r$, that $A /\left(B\left(J_{0}\right) g_{1} \cap \ldots \cap B\left(J_{0}\right) g_{r}\right)$ is a direct sum of certain $A / B\left(J_{0}\right) g$. So we assume that

$$
A /\left(B\left(J_{0}\right) g_{1} \cap \ldots \cap B\left(J_{0}\right) g_{r-1}\right)=\oplus\left\{A / B\left(J_{0}\right) g \mid g=g_{i_{1}}, \ldots, g_{i_{k}}\right\}
$$

If $B\left(J_{0}\right) g_{r} \geq B\left(J_{0}\right) g_{1} \cap \ldots \cap B\left(J_{0}\right) g_{r-1}$, then

$$
A /\left(B\left(J_{0}\right) g_{1} \cap \ldots \cap B\left(J_{0}\right) g_{r}\right)=\oplus\left\{A / B\left(J_{0}\right) g \mid g=g_{i_{1}}, \ldots, g_{i_{k}}\right\}
$$

If $B\left(J_{0}\right) g_{r} \nsucceq B\left(J_{0}\right) g_{1} \cap \ldots \cap B\left(J_{0}\right) g_{r-1}$ then, by the maximality of $B\left(J_{0}\right)$ and hence of $B\left(J_{0}\right) g_{r}$, we have

$$
B\left(J_{0}\right) g_{r}+\left(B\left(J_{0}\right) g_{1} \cap \ldots \cap B\left(J_{0}\right) g_{r-1}\right)=A
$$

and so

$$
A / B_{0}=\oplus\left\{A / B\left(J_{0}\right) g \mid g=g_{i_{1}}, \ldots, g_{i_{k}}, g_{r}\right\}
$$

The first key step in the proof is the following lemma and its corollary which takes the role of Lemma 1 of [7] and Lemma 2 of [1]. 
Lemma 3.2. Let $A$ be a non-zero artinian $\mathbb{Z} G$-module and let $H$ be a normal subgroup of $G$ contained in the $F C$-hypercentre of $G$. If $A=[A, H]$ then there is a normal subgroup $K$ of $G$ and a nonzero $\mathbb{Z} G$-image $\bar{A}$ of $A$ such that $[\bar{A}, K]=\bar{A}, K / C_{K}(\bar{A})$ is finitely generated and $K / C_{K}(\bar{A}) \leq F C\left(G / C_{K}(\bar{A})\right) \cap\left(H C_{K}(\bar{A}) / C_{K}(\bar{A})\right)$.

Proof. By the artinian condition, we may assume that the lemma is true for any proper $\mathbb{Z} G$-submodule $A_{0}$ of $A$ and normal subgroup $H_{0}$ contained in the $F C$ hypercentre of $G$ such that $\left[A_{0}, H_{0}\right]=A_{0}$.

Clearly, by passing to $G / C_{G}(A)$, we may assume that $G$ acts faithfully on $A$.

There is a non-trivial finitely generated normal subgroup $F=<x^{G}>$ contained in $H \cap F C(G)$. If $A=[A, F]$ then we can take $F$ to be the required subgroup and $A$ the required $\mathbb{Z} G$-image.

So we may suppose that $A_{1}=[A, F] \neq A$; since $G$ acts faithfully on $A, A_{1} \neq 0$. Put $G_{1}=C_{G}(F)$ so that $G / H$ is finite and hence $A$ and $A_{1}$ are artinian $\mathbb{Z} G_{1}$-modules. Let $H_{1}=H \cap G_{1}=C_{H}(F)$.

(I) Suppose $\left[A_{1}, H_{1}\right] \neq A_{1}$.

Consider the $\mathbb{Z} G$-module $A^{*}=A /\left[A_{1}, H_{1}\right]$ and its $\mathbb{Z} G$-submodule $A_{1}^{*}=$ $A_{1} /\left[A_{1}, H_{1}\right]$. Now $A_{1}^{*}$ is a trivial $\mathbb{Z} H_{1}$-module and, for each $y \in F$, $A^{*}(1-y) \leq\left[A^{*}, F\right] \leq A_{1}^{*}$. So $A^{*}(1-y)$ is a trivial $\mathbb{Z} H_{1}$-module. As $\mathbb{Z} H_{1}$-modules, $A^{*}(1-y) \cong A^{*} / C_{A^{*}}(\mathrm{y})$ and so $A^{*} / C_{A^{*}}(y)$ is $\mathbb{Z} H_{1}$-trivial, for all $y \in F$. Hence $A^{*} / C_{A^{*}}(F)$ is $\mathbb{Z} H_{1}$-trivial.

The $\mathbb{Z} G$-module $\bar{A}=A^{*} / C_{A^{*}}(F)$ is non-zero; for if $C_{A^{*}}(F)=A^{*}$ then $[A, F] \leq$ $\left[A_{1}, H_{1}\right]$ and so $A_{1}=[A, F]=\left[A_{1}, H_{1}\right]$ contrary to our assumption. Since $[A, H]=A$ we have $\left[A^{*}, H\right]=A^{*}$ and also $[\bar{A}, H]=\bar{A}$. Also $\bar{A}$ is $\mathbb{Z} H_{1}$-trivial and so $H / C_{H}(\bar{A})$ is finite. Therefore in this case we can take $H$ to be the required normal subgroup.

(II) Suppose $\left[A_{1}, H_{1}\right]=A_{1}$.

By our initial assumption about $A$, there is a normal subgroup $K_{1}$ of $G$ and a nonzero $\mathbb{Z} G$-image $\bar{A}_{1}$ of $A_{1}$ such that $\left[\bar{A}_{1}, K_{1}\right]=\bar{A}_{1}, K_{1} / C_{K_{1}}\left(\bar{A}_{1}\right)$ is finitely generated and $K_{1} / C_{K_{1}}\left(\bar{A}_{1}\right) \leq F C\left(G / C_{K_{1}}\left(\bar{A}_{1}\right)\right) \cap\left(H_{1} C_{K_{1}}\left(\bar{A}_{1}\right) / C_{K_{1}}\left(\bar{A}_{1}\right)\right)$.

Let $F=<x_{1}, \ldots, x_{n}>$ and let $D_{i}=A\left(1-x_{i}\right)$, a $\mathbb{Z} G_{1}$-module. Then $A_{1}=$ $[A, F]=D_{1}+\ldots+D_{n}$ and $\bar{A}_{1}=\bar{D}_{1}+\ldots+\bar{D}_{n}$, where $\bar{D}_{i}$ is the image of $D_{i}$ in $\bar{D}$.

Let $k$ be the largest integer such that $\bar{A}_{1}=\bar{D}_{k}+\ldots+\bar{D}_{n}$. Then

$$
\bar{A}_{1} /\left(\bar{D}_{k+1}+\ldots+\bar{D}_{n}\right) \cong \bar{D}_{k} /\left(\bar{D}_{k} \cap\left(\bar{D}_{k+1}+\ldots+\bar{D}_{n}\right)\right) \text {. }
$$

is a $\mathbb{Z} G_{1}$-module which is non-zero by the choice of $k$ and, since $\left[\bar{A}_{1}, K_{1}\right]=\bar{A}_{1}$, we also have

$$
\left[\bar{D}_{k} /\left(\bar{D}_{k} \cap\left(\bar{D}_{k+1}+\ldots+\bar{D}_{n}\right)\right), K_{1}\right]=\bar{D}_{k} /\left(\bar{D}_{k} \cap\left(\bar{D}_{k+1}+\ldots+\bar{D}_{n}\right)\right) .
$$

That is, $D_{k}$ has a nonzero $\mathbb{Z} G_{1}$-image $D_{k}^{*}$ such that $\left[D_{k}^{*}, K_{1}\right]=D_{k}^{*}$. But there is a $\mathbb{Z} G_{1}$-isomorphism

$$
A / C_{A}\left(x_{k}\right) \cong A\left(1-x_{k}\right)=D_{k}
$$

and so $A$ has a nonzero $\mathbb{Z} G_{1}$-image $A / B \cong D_{k}^{*}$ such that $\left[A / B, K_{1}\right]=A / B$.

Now form the $\mathbb{Z} G$-module $B_{0}$ as in Lemma 3.1. Then

$$
B_{0}=\cap_{g \in G} B\left(J_{0}\right) g, B\left(J_{0}\right) \geq B \text { and } A / B_{0}=\oplus\left\{A / B\left(J_{0}\right) g: g=g_{i_{1}}, \ldots, g_{i_{l}}\right\} .
$$


Since $\left[A / B, K_{1}\right]=A / B$, we have $\left[A, K_{1}\right]+B=A$ and so $\left[A, K_{1}\right]+B g=A$, for all $g \in G$. Since $B\left(J_{0}\right) g \geq B g$, it follows that $\left[A / B\left(J_{0}\right) g, K_{1}\right]=A / B\left(J_{0}\right) g$ and, since $A / B_{0}$ is the direct sum of the modules $A / B\left(J_{0}\right) g$, we have $\left[A / B_{0}, K\right]=A / B_{0}$. Write $\bar{A}=A / B_{0}$ so that $\left[\bar{A}, K_{1}\right]=\bar{A}$.

Since $C_{K_{1}}\left(\bar{A}_{1}\right)$ acts trivally on $\bar{A}_{1}$, it also acts trivially on $\bar{D}_{k}$. Since $A / B \cong D_{k}^{*}$, we see that $C_{K_{1}}\left(\bar{A}_{1}\right)$ acts trivially on $A / B$; hence on each $A / B\left(J_{0}\right) g$ and so on $\bar{A}=A / B_{0}$.

Since $C_{K_{1}}(\bar{A})=C_{K_{1}}\left(A / B_{0}\right) \geq C_{K_{1}}\left(\bar{A}_{1}\right)$ and $K_{1} / C_{K_{1}}\left(\bar{A}_{1}\right)$ is a finitely generated subgroup of $F C\left(G / C_{K_{1}}\left(\bar{A}_{1}\right)\right) \cap\left(H_{1} C_{K_{1}}\left(\bar{A}_{1}\right) / \bar{A}_{1}\right)$ we see that $\left.K_{1} / C_{K_{1}}(\bar{A})\right)$ is finitely generated subgroup of $F C\left(G / C_{K_{1}}(\bar{A})\right) \cap\left(H_{1} C_{K_{1}}(\bar{A}) / C_{K_{1}}(\bar{A})\right)$. Therefore we can take $\bar{A}$ and $K_{1}$ to be the required nonzero image and normal subgroup respectively.

The condition that $K / C_{K}(\bar{A})$ is finitely generated can be refined and the following corollary takes this further for the case in which $H$ is locally soluble.

Corollary 3.3. Let $A$ be a non-zero artinian $\mathbb{Z} G$-module and let $H$ be a normal locally soluble subgroup of $G$ contained in the FC-hypercentre of $G$. If $[A, H]=A$ then there is a normal subgroup $K$ of $G$ and a nonzero $\mathbb{Z} G$-image $\bar{A}$ of $A$ such that $[\bar{A}, K]=\bar{A}, K / C_{K}(\bar{A}) \leq F C\left(G / C_{K}(\bar{A})\right) \cap\left(H C_{K}(\bar{A}) / C_{K}(\bar{A})\right)$ and $K / C_{K}(\bar{A})$ is the normal closure of a single element of $G / C_{K}(\bar{A})$ and is either a finite elementary abelian q-group or a free abelian group of finite rank.

Proof. We may replace $A$ by the $\bar{A}$ in the Lemma and $H$ by $K$ and so assume that $H / C_{H}(A) \leq F C\left(G / C_{H}(A)\right), H \triangleleft G,[A, H]=A$ and $H / C_{H}(A)$ is finitely generated. Then $H$ has a finite series of normal subgroups of $G$

$$
C_{H}(A)=H_{0} \leq H_{1} \leq \ldots \leq H_{n}=H
$$

such that $H_{i+1} / H_{i}$ is the normal closure of a single element and is either free abelian of finite rank or finite abelian. Choose $m$ minimal such that $\left[A, H_{m}\right]=A$ and consider the non-zero $\mathbb{Z} G$-image $\bar{A}=A /\left[A, H_{m-1}\right]$. Then $\left[\bar{A}, H_{m}\right]=\bar{A}$ and $C_{H_{m}}(\bar{A}) \geq$ $H_{m-1}$ so that $H_{m} / C_{H_{m}}(\bar{A})$ is the normal closure of a single element and is finitely generated abelian. If $H_{m} / C_{H_{m}}(\bar{A})$ is infinite then there is an integer $t$ such that $K / C_{H_{m}}(\bar{A})=\left\{H_{m} / C_{H_{m}}(\bar{A})\right\}^{t}$ is free abelian. If $[\bar{A}, K]=\bar{A}$, then we are through. If $[\bar{A}, K] \neq \bar{A}$ then consider $\tilde{A}=\bar{A} /[\bar{A}, K]$. We have $\left[\tilde{A}, H_{m}\right]=\tilde{A}$ and $C_{H_{m}}(\tilde{A}) \geq K$, so that $H_{m} / C_{H_{m}}(\tilde{A})$ is finite.

So the only case left to consider is that $A$ has a $\mathbb{Z} G$-image $\tilde{A}$ and $G$ has a normal subgroup $L / C_{L}(\tilde{A}) \leq F C\left(G / C_{L}(\tilde{A})\right)$ such that $[\tilde{A}, L]=\tilde{A}$ and $L / C_{L}(\tilde{A})$ is finite. Now $L$ has a finite series of normal subgroups of $G$

$$
C_{L}(\tilde{A})=L_{0} \leq L_{1} \leq \ldots \leq L_{r}=L
$$

such that $L_{j+1} / L_{j}$ is an elementary abelian group and is the normal closure of a single element of $G / L_{j}$. Choosing $s$ minimal such that $\left[\tilde{A}, L_{s}\right]=\tilde{A}$ we can take $\tilde{A} /\left[\tilde{A}, L_{s-1}\right]$ for the required $\mathbb{Z} G$-image and $L_{s}$ as the required normal subgroup.

Lemma 3.4. Let $A$ be a normal abelian p-subgroup of the group $E$ such that $E / A$ is $F C$-hypercentral and $A$ is artinian as a $\mathbb{Z} E$-module and has no nonzero finite $\mathbb{Z} E$-sections. 
If $N / A$ is a normal subgroup of $E / A$ such that $N \leq C_{E}(A)$, then $N=M \times A$ for some normal subgroup $M$ of $E$. Furthermore, $M$ is contained in any supplement to $A$ in $E$.

Proof. Let $M$ be a normal subgroup of $E$ contained in $N$ maximal subject to $M \cap A=1$. By considering $E / M$ we may assume that $M=1$. Then $E$ satisfies the property

(1) If $S \triangleleft E, S \leq N$ and $S \neq 1$, then $S \cap A \neq 1$.

We show that, in this situation, $A=N$.

Suppose that $A \neq N$. Since $E / A$ is $F C$-hypercentral there is a $K \triangleleft E$ with $A \triangleleft K \leq N$ and $K / A$ finite or free abelian of finite rank and $E / C_{E}(K / E)$ is finite.

If $K / A$ is finite, then $K$ is centre-by-finite and so $K^{\prime}$ is finite. Thus $A \cap K^{\prime}$ is finite and, since $A$ has no finite $\mathbb{Z} E$-submodules, $A \cap K^{\prime}=1$. By condition (1), $K^{\prime}=1$ and so $K$ is abelian. Now $K$ is an artinian $\mathbb{Z} E$-module and so, by Theorem 2.1 , has an $f$ decomposition $K=A \times K^{+}$. But now, applying condition (1) to $K^{+}$, we see that $K^{+}=1$ and so $K=A$, a contradiction.

If $K / A$ is free abelian of finite rank then $K$ is nilpotent of class two. Write $K=<y_{1}, \ldots, y_{n}>Z(K)$ and $\left[y_{i}, y_{j}\right]=a_{i j} \in A$. There is an integer $k$ such that $a_{i j}^{p^{k}}=1$, for all $i, j$, and so $\left[y_{i}^{p^{k}}, y_{j}\right]=1$, for all $i, j$, and so $y_{i}^{p^{k}} \in Z(K)$. Therefore $K / Z(K)$ is finite and so $K^{\prime}$ is finite. Again $A \cap K^{\prime}=1$ and, by condition (1), $K^{\prime}=1$. That is, $K$ is abelian.

Since $A$ is artinian as a $\mathbb{Z} E$-module we can choose a normal subgroup $D$ of $E$ contained in $K$ such that $D / D \cap A$ is infinite and $D \cap A$ is minimal with this property.

By condition (1), $D \cap A \neq 1$. Since $D \cap A$ has no finite $\mathbb{Z} E$-submodules, $E / C_{E}(D)$ must be infinite but $E_{1}=C_{E}(D / D \cap A)$ has finite index in $E$. Therefore there is a nontrivial normal subgroup $X / C_{E}(D)$ of $E / C_{E}(D)$ such that $X \leq E_{1}, X$ is either finite or free abelian of finite rank and $E / C_{E}\left(X / C_{E}(D)\right)$ is finite. Let $E_{2}=E_{1} \cap C_{E}\left(X / C_{E}(D)\right)$ so that $E / E_{2}$ is finite.

For $x \in X \backslash C_{E}(D), D(1-x) \leq D \cap A$ and $D(1-x) \neq 0$. Now $D(1-x)$ has no nonzero finite $\mathbb{Z} E_{2}$-sections; but $D(1-x) \cong D / C_{D}(x)$ as $\mathbb{Z} E_{2}$-modules, so that $D / C_{D}(x)$ is a nonzero $\mathbb{Z} E_{2}$-image of $D$ with no nonzero finite $\mathbb{Z} E_{2}$-sections.

Let $X / C_{E}(D)=C_{E}(D)<x_{1}, \ldots, x_{m}>$; then $C_{D}(X)=C_{D}\left(x_{1}\right) \cap \ldots \cap C_{D}\left(x_{m}\right)$ and so $D / C_{D}(X)$ has no nonzero finite $\mathbb{Z} E_{2}$-sections. Since $D / D \cap A$ is a finitely generated abelian group we have $C_{D}(X)+(D \cap A)=D$ and hence $C_{D}(X)+A=$ $D+A$. But $C_{D}(X) \triangleleft E$ and $C_{D}(X) \cap A \neq D \cap A$ giving a contradiction to the choice of $D$.

This completes the proof that, under condition (1), $A$ must be equal to $N$ and so under the hypotheses of the lemma we have shown the existence of an $M \triangleleft E$ such that $N=M \times A$.

Finally, let $S$ be a supplement to $A$ in $E$; that is, $S A=E$. Then $N=(S \cap N) A$ and $S \cap N \triangleleft S A=E$. Now

$$
M(S \cap N) /(S \cap N) \leq N / S \cap N=A(S \cap N) /(S \cap N)
$$

and so $M(S \cap N) /(S \cap N)$ has no finite $\mathbb{Z} E$-section. But $M(S \cap N) /(S \cap N) \cong$ $M /(M \cap S)$ which does have finite $\mathbb{Z} E$-sections if it is non-zero. Therefore $M=M \cap S$, as required. 
The following two lemmas give partial splitting and conjugacy results. They are well known in various forms but we give details in order to state the versions we need and also to indicate the reasons for the lemmas which follow.

Lemma 3.5. Suppose that $A$ is a $\mathbb{Z} H$-module, $<x>$ is a central cyclic subgroup of $H$ such that $A(1-x)=A$ and let $E$ be any extension of $A$ by $H$. Then for any $\mathbb{Z} H$ submodule $B$ of $A$ containing $C_{A}(x), E / B$ splits over $A / B$.

Proof. Let $g \in E$ and let $y$ be an inverse image of $x$ in $E$; then $\left(y^{-1}\right)^{g}=a y^{-1}$, for some $a \in A$. Since $A(1-x)=A$, there is an element $b \in A$ such that $a=b y^{-1} b^{-1} y$ and so $\left(y^{-1}\right)^{g}=a y^{-1}=\left(y^{-1}\right)^{b^{-1}}$. Therefore $g b \in C_{E}(y)$ and $g \in A C_{E}(y)$. Since $C_{E}(y) \cap A=C_{A}(y)=C_{A}(x)$ we see that $C_{E}(y) / C_{A}(x)$ is a complement to $A / C_{A}(x)$ in $E / C_{A}(x)$. It is now clear that if $B \geq C_{A}(x)$ then $B C_{E}(y) / B$ is a complement to $A / B$ in $E / B$.

Lemma 3.6. Suppose that $A$ is a $\mathbb{Z} H$-module, $<x>$ is a central cyclic subgroup of $H$ such that $A(1-x)=A$ and let $E$ be a split extension of $A$ by $H$. If $K$ is a second complement to $A$ in $E$ and if $B$ is any $\mathbb{Z} H$-submodule of $A$ containing $C_{A}(x)$, then $H B$ and $K B$ are conjugate in $E$.

Proof. It is clear that $A<x>\cap H=<x>$ and that $A<x>\cap K$ is generated by an element $a x^{-1}$, for some $a \in A$. Thus $K \leq C_{E}\left(a x^{-1}\right)$ and $C_{E}\left(a x^{-1}\right)=K C_{A}(x)$. Also $C_{E}(x)=H C_{A}(x)$.

Since $A(1-x)=A$, there is an element $b \in A$ such that $a=b x^{-1} b^{-1} x$ and so $a x^{-1}=b x^{-1} b^{-1}$. It follows that

$$
K C_{A}(x)=C_{E}\left(a x^{-1}\right)=C_{E}\left(b x^{-1} b^{-1}\right)=b\left(H C_{A}(x)\right) b^{-1}
$$

If $B$ contains $C_{A}(x)$, then $K B=b(H B) b^{-1}$, as required.

Our first step towards making use of these two lemmas is to obtain a suitable $\mathbb{Z} H$-image $\bar{A}$ such that $\bar{A}(1-x)=\bar{A}$.

Lemma 3.7. Let $A$ be an artinian $\mathbb{Z} H$-module and $x \in Z(H)$ such that $x$ does not act polytrivially on $A$. Then there is a nonzero $\mathbb{Z} H$-image $\bar{A}=A / B$ such that $\bar{A}(1-x)=\bar{A}$ and $B \geq C_{A}(x)$.

Proof. There is a descending chain of $\mathbb{Z} H$-submodules

$$
A \geq A(1-x) \geq A(1-x)^{2} \geq \ldots
$$

Since $A$ is artinian, there is a least integer $n$ such that $A(1-x)^{n}=A(1-x)^{n+1}$ and, since $x$ does not act polytrivially, $A(1-x)^{n} \neq 0$. We prove the result by induction on this integer $n$, the result being trivially true if $n=0$.

Write $A_{i}$ for $A(1-x)^{i}$. Then

$$
A_{n}=A_{n-1}(1-x) \cong A_{n-1} / C_{A_{n-1}}(x) \text {. }
$$


Consider $A / C_{A_{n-1}}(x)$ and write $A_{i}^{*}$ for $A_{i} / C_{A_{n-1}}(x)$. Then $x$ does not act polytrivally on $A_{0}^{*}, A_{i}^{*}=A_{0}^{*}(1-x)^{i}$ and $A_{n-1}^{*}(1-x)=A_{n-1}^{*}$. By induction, $A / C_{A_{n-1}}(x)$ has a nonzero $\mathbb{Z} H$-image $\bar{A}=A / B$ such that $\bar{A}(1-x)=\bar{A}$ and $B / C_{A_{n-1}}(x) \geq$ $C_{A_{0}^{*}}(x) \geq C_{A}(x) / C_{A_{n-1}}(x)$. Therefore $B \geq C_{A}(x)$.

In the situations where we apply Lemmas 3.5 to 3.7 , the group $H$ will be the centralizer $G_{1}$ of some normal subgroup $\left\langle x^{G}>\right.$, so that $G / G_{1}$ will be finite. Lemma 3.7 will give us a $\mathbb{Z} G_{1}$-image of $A$ in which we can apply Lemmas 3.5 and 3.6, but we will need a $\mathbb{Z} G$-image. This will be provided by the construction given in Lemma 3.1.

The direct sum constructed in that lemma will be used in a number of places in the main proof to give the existence and conjugacy of complements in certain sections $E_{1} / B_{0}$ where $E / E_{1}$ is finite and $B_{0} \triangleleft E$. The simple step of moving from $E_{1} / B\left(J_{0}\right)$ with $B\left(J_{0}\right) \triangleleft E_{1}$ to $E_{1} / B_{0}$ is given in the next lemma.

Lemma 3.8. Let $A$ be a normal subgroup of the group $E$ and suppose that $A$ contains subgroups $A_{i} \triangleleft E$ with $A=D r_{i=1}^{n} A / A_{i}$.

(i) If $A / A_{i}$ has a complement $S_{i} / A_{i}$ in $E$, for each $i$, then $A$ has a complement $S$ in $E$.

(ii) If $S$ and $T$ are complements to $A$ in $E$ such that $S A_{i}$ is conjugate to $T A_{i}$, for each $i$, then $S$ and $T$ are conjugate in $E$.

Proof. (i) Let $A_{1} \cap \ldots \cap A_{r-1}=B$ and assume, inductively, that $A / B$ has a complement $R / B$ in $E / B$. Consider $R \cap S_{r}$. Certainly $R \cap S_{r} \cap A=B \cap A_{r}$. Also $\left(R \cap S_{r}\right) A=\left(R \cap S_{r}\right) B A_{r}=\left(R \cap S_{r} B\right) A_{r}=\left(R \cap S_{r} A\right) A_{r}=R A_{r}=R B A_{r}=R A=E$. Therefore $R \cap S_{r} / A_{r} \cap B$ is a complement in $E / A_{1} \cap \ldots \cap A_{r}$.

(ii) Again writing $B=A_{1} \cap \ldots \cap A_{r-1}$ we assume inductively that $S B$ and $T B$ are conjugate. Replacing $T$ by an appropriate conjugate we may assume that $S B=T B$. There is an element $a \in A$ such that $S^{a} A_{r}=T A_{r}$. Since $A=B A_{r}$ we may assume that $a \in B$ and so $S^{a} B=T B$. Now $T\left(A_{r} \cap B\right)=T\left((T \cap A) A_{r} \cap B\right)=$ $T\left(T A_{r} \cap B\right)=T A_{r} \cap T B$. So $\quad T\left(A_{r} \cap B\right)=S^{a} A_{r} \cap S B=S^{a}\left(A_{r} \cap B\right) \quad$ and $\quad$ so $T\left(A_{1} \cap \ldots \cap A_{r}\right)$ is conjugate to $S\left(A_{1} \cap \ldots \cap A_{r}\right)$, as required.

TheOREM 3.9. Let $G$ be a locally soluble FC-hypercentral group and $A$ a periodic artinian $\mathbb{Z} G$-module with no non-zero finite $\mathbb{Z} G$-submodules. Then any extension $E$ of $A$ by $G$ splits conjugately over $A$.

\section{Proof. Existence of complements.}

Since $A$ is artinian, $E$ has a subgroup $E_{0}$ minimal with respect to $E=A E_{0}$. We prove that $A \cap E_{0}=1$.

Suppose, if possible, that $A_{0}=A \cap E_{0} \neq 1$. The group $E_{0}$ and its subgroup $A_{0}$ considered as a $\mathbb{Z} G$-module satisfy the conditions of the lemma. We may therefore assume that $A=A_{0}, E=E_{0}$ so that

(2) $A$ has no proper supplement in $E$.

Under this extra hypothesis we shall obtain a contradiction.

Clearly, we may assume that $A$ is a $p$-group. Since $A$ has no finite $\mathbb{Z} G$-sections, it follows that $[A, G]=A$. By Corollary 3.3 , there is a non-zero $\mathbb{Z} G$-image $\bar{A}=A / B$ of $A$ and a nontrivial normal subgroup $K / A, K / C_{K}(\bar{A}) \leq F C\left(E / C_{K}(\bar{A})\right), K / C_{K}(\bar{A})$ is the normal closure of a single element $\bar{x}$ in $\bar{E}=E / C_{K}(\bar{A})$ such that $[\bar{A}, K]=\bar{A}$ and is 
either a finite elementary abelian $q$-group or a free abelian group of finite rank. Passing to the factor group $E / B$, we can take $B=1$, so that $[A, K]=A$.

(I) $K / C_{K}(A)$ is a finite abelian q-group.

By Lemma 3.4, $C_{K}(A)=M \times A$, for some $M \triangleleft E$. Passing to the factor group $E / M$, we can take $M=1$ and so assume that $C_{K}(A)=A$ and $K / A$ is a finite abelian $q$-group. (2).

If $q=p$, then by Lemma 4 of [7], $A$ has a proper supplement in $E$, contrary to

If $q \neq p$, then $K=A Q$, where $Q$ is a finite Sylow $q$-subgroup of $K$ and the Sylow $q$-subgroups of $K$ are conjugate. The Frattini argument shows that $E=A N_{E}(Q)$ and so, by $(2), N_{E}(Q)=E$ and $[A, Q]=1$, contrary to $C_{K}(A)=A$.

(II) $K / C_{K}(A)$ is free abelian of finite rank.

Again we may apply Lemma 3.4 to assume that $C_{K}(A)=A$ so that $K / A$ is free abelian of finite rank and $K / A=<x A>E / A$.

Let $E_{1}=C_{E}(K / A)$ so that $E / E_{1}$ is finite. Thus $A$ is an artinian $\mathbb{Z} E_{1}$-module and has no non-zero finite $\mathbb{Z} E_{1}$-sections. Write $x A=\bar{x} \in G_{1}=E_{1} / A$ and let $\left\{\bar{x}^{G}\right\}=\left\{\bar{x}=x_{1}, x_{2}, \ldots, x_{n}\right\}$ with $x_{i}=\bar{x}^{g_{i}}$. If $x$ acts polytrivially on $A$ then so does each $x_{i}$. Hence $A\left(1-x_{i}\right) \neq A$ and, inductively, $A\left(1-x_{1}\right)+\ldots+A\left(1-x_{r-1}\right) \neq A$. But then $x_{r}$ acts polytrivially on $A / A\left(1-x_{1}\right)+\ldots+A\left(1-x_{r-1}\right)$ and so $A\left(1-x_{1}\right)$ $+\ldots+A\left(1-x_{r}\right) \neq A$. It follows that $[A, K]=A\left(1-x_{1}\right)+\ldots+A\left(1-x_{n}\right) \neq A$, contrary to our definition of $K$. Therefore $x$ does not act polytrivially on $A$ and so, by Lemma $3.6, A$ has a nonzero $\mathbb{Z} E_{1}$-image $\bar{A}=A / B$ such that $\bar{A}(1-x)=\bar{A}$ and $B \geq C_{A}(x)$.

Now form the $\mathbb{Z} E$-submodule $B_{0}$ as in Lemma 3.7. Then $B_{0}=\cap_{g \in G} B\left(J_{0}\right)^{g}$, $B\left(J_{0}\right) \geq B \geq C_{A}(x)$ and $A / B_{0}=\oplus\left\{A / B\left(J_{0}\right)^{g} \mid g=g_{i_{1}}, \ldots, g_{i_{l}}\right\}$. Note also that $B\left(J_{0}\right)^{g} \geq B^{g} \geq C_{A}\left(x^{g}\right)$.

By Lemma 3.4, $E_{1} / B\left(J_{0}\right)^{g}$ splits over $A / B\left(J_{0}\right)^{g}$. By Lemma 3.8, there is a complement $L / B_{0}$ to $A / B_{0}$ in $E_{1} / B_{0}$.

Since $B\left(J_{0}\right) \geq B$, we have $\left(A / B\left(J_{0}\right) 1-x\right)=A / B\left(J_{0}\right)$ and so $C_{A / B\left(J_{0}\right)}(x)$ $\neq A / B\left(J_{0}\right)$. Define the $\mathbb{Z} E_{1}$-submodule $C$ by $C / B\left(J_{0}\right)=C_{A / B\left(J_{0}\right)}(x)$. Then $C^{g} / B\left(J_{0}\right)^{g}=C_{A / B\left(J_{0}\right)^{g}}\left(x^{g}\right)$ so that $C^{g} / B_{0} \geq C_{A / B_{0}}\left(x^{g}\right)$. Let $C_{0}=\cap_{g \in G} C^{g}$; then $C_{0}$ is a $\mathbb{Z} E$-submodule and $A / C_{0}=\oplus\left\{A / C^{g} \mid g=g_{i_{1}}, \ldots, g_{i_{l}}\right\}$.

Let $L$ and $M$ be two complements to $A / B_{0}$ in $E_{1} / B_{0}$. By Lemma $3.5, L C^{g}$ and $M C^{g}$ are conjugate in $E_{1}$. It now follows from Lemma 3.8(ii) that $L C_{0}$ and $M C_{0}$ are conjugate in $E_{1}$.

The Frattini argument now shows that $E=A N_{E}\left(L C_{0}\right)$. Since $A$ has no proper supplement in $E$, we must have $L C_{0} \triangleleft E$. Hence $\left[A, L C_{0}\right] \leq A \cap L C_{0}=$ $(A \cap L) C_{0}=C_{0} \neq A$, contrary to $[A, K]=A$.

This completes the proof that $E$ splits over $A$.

Conjugacy of complements

Let $L_{1}$ and $L_{2}$ be two complements to $A$ in $E$. Since $A$ is artinian there is a normal subgroup $A_{0}$ of $E$ contained in $A$ such that $L_{1} A_{0}$ and $L_{2} A_{0}$ are conjugate but for any normal subgroup $A_{1}$ of $E$ properly contained in $A_{0}, L_{1} A_{1}$ and $L_{2} A_{1}$ are not conjugate. We may assume that $L_{1} A_{0}=L_{2} A_{0}, E=L_{1} A_{0}$ and $A=A_{0}$, so that we have

(3) $L_{1} A=L_{2} A=E$ and if $A_{1}$ is a normal subgroup of E properly contained in $A$, then $L_{1} A_{1}$ and $L_{2} A_{1}$ are not conjugate in $A_{1}$.

By Corollary 3.3 , there is a non-zero $\mathbb{Z} G$-image $\bar{A}=A / B$ of $A$ and a normal subgroup $K / A$ of $E / A$ such that $[\bar{A}, K]=\bar{A}$ and $K / C_{K}(\bar{A})=<\bar{x}^{\bar{G}}>$ is a nontrivial 
finite abelian $q$-group or is free abelian of finite rank. Passing to the factor group $E / B$ we can take $B=1$ so that $[A, K]=A$.

(I) $K / C_{K}(A)$ is a finite abelian q-group.

By Lemma 3.4, $C_{K}(A)=M \times A$ for some $M \triangleleft E$ and $M$ is contained in $L_{1}$ and $L_{2}$. We may therefore pass to the factor group $E / M$ and assume that $C_{K}(A)=A$ so that $K / A$ is a finite abelian $q$-group.

If $q=p$, then by Lemma 4 of [7], $L_{1}$ and $L_{2}$ are conjugate modulo some proper $E$-invariant subgroup of $A$, contrary to (3).

If $q \neq p$, then $L_{1} \cap K$ and $L_{2} \cap K$ are finite Sylow $q$-subgroups of $K$ and so are conjugate in $K$. By the Frattini argument, $E=A N_{E}\left(L_{i} \cap K\right)$ and $A \cap N_{E}\left(L_{i} \cap K\right)=C_{A}\left(L_{i} \cap K\right)=C_{A}(K)$. By Fitting's Lemma, $A=C_{A}(K) \oplus[A, K]$ and since $[A, K]=A, \quad C_{A}(K)=1$. Therefore $A \cap N_{E}\left(L_{i} \cap K\right)=1$ and, since $L_{i} \leq N_{E}\left(L_{i} \cap K\right)$ we must have $L_{i}=N_{E}\left(L_{i} \cap K\right)$. Since $L_{1} \cap K$ and $L_{2} \cap K$ are conjugate, it follows that $L_{1}$ and $L_{2}$ are conjugate.

(II) $K / C_{K}(A)$ is free abelian of finite rank.

Again we may apply Lemma 3.4 to assume that $C_{K}(A)=A$, so that $K / A=<x A>^{E / A}$ is free abelian.

Let $E_{1}=C_{E}(K / A)$ so that $E / E_{1}$ is finite and $E_{1} \cap L_{i}$ is a complement to $A$ in $E_{1}$.

As in the existence part of the proof, $x$ does not act polytrivially on $A$ and so there is a non-zero $\mathbb{Z} E_{1}$-image $\bar{A}=A / B$ of $A$ such that $\bar{A}(1-x)=\bar{A}$ and $B \geq C_{A}(x)$. We form the $\mathbb{Z} E$-submodule $B_{0}$ as in Lemma 3.1 so that $B_{0}=\cap_{g \in E} B\left(\bar{J}_{0}\right)^{g}$ and $A / B_{0}=\oplus\left\{A / B\left(J_{0}\right)^{g} \mid g=g_{i_{1}}, \ldots, g_{i_{l}}\right\}$ with $B\left(J_{0}\right) \geq B \geq C_{A}(x)$.

Then, for each $g \in E, B\left(J_{0}\right)^{g} \geq C_{A}\left(x^{g}\right)$ and so, by Lemma 3.6, $\left(E_{1} \cap L_{1}\right) B\left(J_{0}\right)^{g}$ is conjugate to $\left(E_{1} \cap L_{2}\right) B\left(J_{0}\right)^{g}$. By Lemma 3.8(ii), $\left(E_{1} \cap L_{1}\right) B_{0}$ is conjugate to $\left(E_{1} \cap L_{2}\right) B_{0}$ in $E_{1}$.

But $\left(E_{1} \cap L_{i}\right) B_{0}=E_{1} \cap L_{i} B_{0} \quad$ and $\quad L_{i} B_{0} \leq N_{E}\left(E_{1} \cap L_{i} B_{0}\right)$. Therefore $N_{E}\left(E_{1} \cap L_{i} B_{0}\right)=L_{i} N_{A}\left(\left(E_{1} \cap L_{i}\right) B_{0}\right)$. But $N_{A}\left(\left(E_{1} \cap L_{i}\right) B_{0}=C_{A / B_{0}}\left(E_{1}\right)\right.$ and since $A$ has no non-zero finite $\mathbb{Z} E_{1}$-section, it follows that $N_{A}\left(\left(E_{1} \cap L_{i}\right) B_{0}\right)=B_{0}$ and so $N_{E}\left(E_{1} \cap L_{i} B_{0}\right)=L_{i} B_{0}$. Therefore $L_{1} B_{0}$ and $L_{2} B_{0}$ are conjugate, contrary to (3).

This completes the proof of the main theorem.

Two questions concerning this result remain open. As with the results on abelian-by-hyperfinite groups we have assumed that the module $A$ is a periodic abelian group and also that the group $G$ is locally soluble. It would also be more satisfactory if this type of result could be fitted into the homological setting of [4].

\section{REFERENCES}

1. Z. Y. Duan, Extensions of abelian-by-hyper (cyclic or finite) groups, I, Comm. Algebra 20 (1992), 2305-2321.

2. Z. Y. Duan, Extensions of abelian-by-hyper (cyclic or finite) groups, II, Rend. Sem. Mat. Univ. Padova 89 (1993), 113-126.

3. L. A. Kurdachenko, B. V. Petrenko and I. Ya. Subbotin, On generalized hypercentres in artinian modules, Comm. Algebra 25 (1997), 1023-1046.

4. D. J. S. Robinson, The vanishing of certain homology and cohomology groups, $J$. Pure Appl. Algebra 7 (1976), 145-167.

5. D. I. Zaicev, Hypercyclic extensions of abelian groups, Akad. Nauk. Ukrain. SSR, Inst. Mat. Kiev (1979), 16-37.

6. D. I. Zaicev, Extensions of abelian groups, Akad. Nauk. Ukrain. SSR, Inst. Mat. Kiev (1980), 16-40. 
7. D. I. Zaicev, Splittable extensions of abelian groups, Akad. Nauk. Ukrain. SSR, Inst. Mat. Kiev (1986), 21-31.

8. D. I. Zaicev, Hyperfinite extensions of abelian groups, Akad. Nauk. Ukrain. SSR, Inst. Mat. Kiev (1988), 17-26.

9. M. J. Tomkinson, Abelian-by-FC-hypercentral groups, Ukrain. Mat. Zh. 43 (1991), $1038-1042$. 